\title{
BACTERIOLOGICAL, CHEMICAL, AND NUTRITIONAL STUDIES OF DENTAL CARIES BY THE MICHIGAN RESEARCH GROUP1
}

\author{
A SUMMary \\ RUSSELL W. BUNTING, D.D.S., D.D.Sc., F.A.C.D. \\ School of Dentistry, University of Michigan, Ann Arbor, Mich.
}

The investigations which have been carried on by the Michigan group during the past four years, in the study of dental caries, have been directed along three general lines: bacteriology and immunology, chemistry of blood and saliva, and nutrition. Each project has been reported in detail in special communications, which have already been published or are in press. Since these reports are appearing in a variety of professional journals - dental, bacteriological, chemical, and nutritional - it has seemed desirable to prepare a brief summary of the entire group of publications for the convenience of those who are interested in the study of the whole problem.

Bacteriologic studies. Jay et al. (1), in a comprehensive review of their studies on the bacteriology of dental caries, reaffirm the statement they and others made previously that a close correlation exists between the activity of B. acidophilus in the mouth and dental caries. They state that when growth of this organism is consistently prolific in the mouth, caries invariably follows; and that when there is no dental disease, B. acidophilus is either consistently absent from the mouth and intestinal tract, or is present only in small numbers, or appears intermittently. They were unable to implant human strains of $B$. acidophilus in the mouths of caries-free rats, or in the mouths or intestinal tracts of humans who were immune to dental caries. A group of caries-free children were fed a pint of acidophilus milk daily

\footnotetext{
1 Researches conducted in the School of Dentistry of the University of Michigan, under the auspices of the Children's Fund of Michigan. Presented at the twelfth general meeting of the International Association for Dental Research, Chicago, Ill. March $18-19,1934$.
} 
over a period of one week. At the end of this period the organism could not be recovered from the mouth, but was present in the intestinal tract. Two weeks later cultures of the stools were also negative. These observations strongly suggest the existence of an immunologic influence against the growth of B. acidophilus in caries-free individuals. In a study of the immunologic factors involved, the presence of agglutinins against B. acidophilus was demonstrated in the blood serum of caries-free patients. Furthermore, these investigators were able to raise the $B$. acidophilus agglutinin titre of blood-serum from caries-susceptible patients by injection of vaccine containing $\mathbf{B}$. acidophilus. In skin tests, made by intradermal injection of $\mathrm{B}$. acidophilus filtrates, it was found that many caries-susceptible individuals gave a positive reaction, while the caries-free were usually negative (2). Although the correlation of these tests to caries susceptibility was not 100 percent, the frequency of correspondence strongly indicates that some relationship exists. These findings substantiate the view that dental caries is a specific bacterial disease, and indicate the existence of controlling immunologic factors which might constitute a means of preventing the disease.

Intensive studies of the typal forms of oral and intestinal B. acidophilus have been made by Faith Hadley, et al. $(3,4)$, in which they have found that no consistent differences exist between the oral and intestinal strains. They have shown close correlation of this organism in the mouth to the activity of dental caries, and have determined the frequency of occurrence of various types of $B$. acidophilus under different degrees of caries susceptibility.

A valuable aid in the study of the bacteriology of dental caries was furnished by Faith Hadley (5) in the development of a method for quantitative estimation of $\mathrm{B}$. acidophilus in saliva. Although this method does not give results that are as uniform as might be desired, owing largely to difficulties in obtaining uniform samples from the mouth, it does afford a useful selective method to cultivate B. acidophilus. Very illuminating data on the activity of this organism in the mouth, and its relationship to degree of caries activity, have been revealed by its use. In cases in which $B$. acidophilus was shown by the qualitative test to be present in caries-immune mouths, almost invariably the quantitative method indicated that the number of these 
organisms was so small as to be insignificant, and that their presence was not constant. In a study of fourteen caries-susceptible children it was found that quantitative estimations of $B$. acidophilus in saliva, made over a period of several months, ranged from a few hundred to 500,000 per cc.; average, 60,000. A similar study of ten caries-free children showed that many of them were negative to $B$. acidophilus, and that in 81 percent of cases the B. acidophilus count was less than 100 per cc. of saliva. A few counts ranged higher. In a Negress, the count rose to a maximum of 19,200 , but this was transitory and later dropped to zero. The average of sixty-nine examinations, including the exceptionally high counts, was 600 .

These data confirm the view that dental caries is a bacteriologic disease, and that B. acidophilus is the most important specific etiologic factor. The growth of this organism in the mouth, and the degree of its caries-producing activity, are determined by certain underlying conditions of natural bacterial immunity and by dietary considerations which at the present time are not fully understood.

Chemical studies. Intensive studies have been made of chemical constituents of saliva and blood-serum as they relate to caries susceptibility. Hubbell has reported (6) data obtained from an 18-months study of saliva of 102 public school children, ranging in ages from 7 to 16 years. In these studies no relationship could be found between the calcium $(\mathrm{Ca})$ and phosphorus $(\mathrm{P})$ content of saliva and the occurrence of dental caries. To a group of these children one quart of milk and two ounces of tomato juice were given daily for eleven months; some also received viosterol. Although a slight decrease was noted in the frequency of dental caries among those receiving these dietary supplements, there was no consistent change in salivary $\mathrm{Ca}$ or P.

Analyses were also made by Hubbell ( 7$)$ of saliva and blood serum from fifteen public-school children who were free from caries, and seventeen who had active caries. These children ranged in age from 9 to 16 years. Salivary analyses, made over a period of eighteen months, included estimations of total solids and ash; also $\mathrm{Ca}, \mathrm{P}, \mathrm{Cl}$, diastatic activity, $\mathrm{pH}, \mathrm{CO}_{2}$-capacity, and total alkalinity. There were no consistent differences between the two groups in the amounts of these constituents, with two possible exceptions. It was found 
that the average salivary $\mathrm{CO}_{2}$-capacity of caries-free children was about 44 percent higher than that of children of the same age who had active caries. The values obtained from many individuals in both groups, however, varied widely from the averages. The average titratable alkalinity of saliva from the caries-free children was 24 percent higher than that for the caries-susceptible group. Individual exceptions to this rule also occurred in both groups. These differences were not found in the blood serum.

In a study by Koehne (10), in which a group of children whose quantitative food-intake over a period of months was satisfactory nutritionally, it was found that no constant correlation existed between alkalinity of saliva and either acid-base value of the diet or activity of dental caries. She found that alkalinity of saliva was not raised by increasing the amount of basic constituents of the diet, and that in caries-arrested children saliva was not consistently higher in $\mathrm{CO}_{2}$-capacity. It is, therefore, the opinion of the group that freedom from dental caries is not related to increased alkalinity of saliva.

Hubbell and Koehne (8) made N, Ca, and $\mathrm{P}$ balance determinations on seventeen children, 7 to 11 years old, covering 35 week-collections. The only variable was the amount of sugar eaten. In those having from 1 to 11 percent of the caloric intake in the form of sugar, no change was noted in the retention of these elements; but in those who received 18 percent additional calories in the form of sugar, there were notable increases in retention of $\mathrm{N}$ and $\mathrm{P}$; $\mathrm{Ca}$ retention was decreased in five out of seven cases. In cases showing evident arrest of dental caries, there was no such change in $\mathrm{Ca}$ and $\mathrm{P}$ retention as has recently been reported by Boyd and Drain (9).

The chemical studies by this group on saliva and blood from cariesfree and caries-susceptible individuals, therefore, do not support claims made by others that the occurrence or degree of activity of dental caries is related to $\mathrm{Ca}, \mathrm{P}$, or acid-neutralizing values of either blood or saliva. No chemical differences were found in any other blood or salivary constituents which could be related to dental disease.

Nutritional studies. Studies of nutrition in relation to dental caries, conducted by Koehne and Morrell, were confined to three projects: (1) dietary supervision of 23 children in the University Hospital, Ann 
Arbor, Michigan; (2) dietary study of 169 children in an orphan asylum; and (3) study of food habits of 9 caries-free and 9 caries-susceptible public-school children. These studies have been continued over periods of one to three years.

The study of the hospital group (10) was made on 23 metabolically normal girls from 6 to 13 years of age, all of whom were placed on a basal diet that was adequate nutritionally. Some were continued on this diet throughout the period of observation. Others were given, also, 100 grams of sugar daily in the form of candy. Some were given candy before meals; others, after meals. There was considerable variation in the food regimen, and the children were afforded freedom of choice in extra helpings of available foods. All foods, eaten, were weighed and recorded. Neither cod-liver oil nor sun-lamp treatment was given. Observation periods ranged from 5 to 18 months. As reported by Koehne and Morrell (11), intake of the various types of foods was remarkably constant for each child, regardless of the amount of candy eaten. Any change, therefore, which occurred in caries activity in these children while on this diet could not be related to differences in $\mathrm{Ca}, \mathrm{P}$, or vitamin intake, for these were constant. Of the 14 children continuously on the basal diet, or on this diet plus a moderate amount of sugar, 9 showed evidence of arrested caries; 5 had very little caries. Arrest of caries was associated with disappearance, or a very low count, of $\mathbf{B}$. acidophilus in the saliva, while the counts remained high in the 5 cases showing continued caries activity. Among the 13 children on the basal diet plus a high sugar content, 9 had active and extensive caries. They also showed marked increase in the B. acidophilus content of the saliva. Three of the remaining children were true caries-immunes. There was no apparent difference between those who ate candy before meals and those who ate it after meals. When a diabetic diet was fed to a highly susceptible individual having a high $B$. acidophilus count, there was marked reduction of these organisms, approaching or reaching zero within a few days. In none of these cases was any change noted in salivary $\mathrm{Ca}, \mathrm{P}$, or acidneutralizing power which could be related to the changed caries susceptibility. There was, however, an 88.9 percent correlation between activity of $\mathrm{B}$. acidophilus in the saliva and dental findings for the whole group. 
In the study of the orphanage group, as reported by Koehne (12), 169 children were observed over periods of from one to four and onehalf years. This group was selected because all children in this institution, numbering about 300 , had been examined by members of the Research Group at least once a year during the preceding six years, and found to have a remarkably low degree of caries. From 75 to 80 percent were entirely caries-free, and only 5 to 6 percent had an appreciable amount of dental disease. A study of the dietary conditions of these children was, therefore, undertaken to determine whether any apparent nutritional factor could be related to the freedom from caries. It was found that the diet, which had been fairly uniform throughout the period of observation, did not meet standard requirements for adequate nutrition. It was below the minimum standard requirement for calories in each age-group, and also inadequate in $\mathrm{Ca}, \mathrm{P}$, and vitamin-D contents. It contained little milk and no butter. A small amount of meat was served daily. No oranges were available except at Christmas, but various vegetables were provided throughout the year, many of which were fed raw. Dessert consisted mostly of raw apple. Cookies and sweet dessert were fed only on Sunday. Of the total caloric intake, 40 to 45 percent was in the form of starch and cereals. No candy was allowed at any time. Only minimum amounts of sugar were used to sweeten foods, and no sugar was placed on the table. Certainly the freedom from caries among these children could not be attributed to adequacy of diet, nor correlated with any consistent increase in $\mathrm{CO}_{2}$-content of saliva, nor to intake of $\mathrm{Ca}, \mathrm{P}$, or vitamin $\mathrm{D}$. There was, however, an 88 percent correlation with the $\mathrm{B}$. acidophilus content of saliva. No conclusions could be drawn from these studies relative to influence of nutrition on the freedom from dental caries among these children. Only three principles were noted that might possibly have a bearing: (1) low sugar content; (2) uniformity and regularity of diet; and (3) hard fibrous raw-fruit eaten at the end of meals.

In the third nutritional study, reported by Koehne (13), an analysis was made of food habits and dietary intake of 9 caries-free and 9 caries-susceptible children in public schools. These children were selected as the most typical cases obtainable in the two groups. Certain distinctive differences were found. Almost without excep- 
tion those in the caries-free group had excellent appetites, and were not notional in food selections; as a rule, their meals were regular and uniform in character; one could take no milk because of allergy; they ate candy and simple desserts occasionally, but had no craving for sweets. The caries-susceptible group were decidedly notional in their food preferences, and did not select uniform or balanced rations; 3 had little milk -2 had one quart per day; practically all craved sweets, and ate considerable quantities of candy and highly sweetened foods. The chemical findings are included in the report of Hubbell (7) in which it is stated that no consistent difference could be found in either saliva or blood from the two types of individuals. A very clear correlation between salivary content of B. acidophilus and caries-susceptibility was shown by Hadley (5).

In view of many current statements relative to the importance of various food elements as protectives against dental caries, the results of the nutritional studies were most interesting. The fact that dental caries was completely arrested in a large majority who received, over a long period of time, a diet that was low in $\mathrm{Ca}$, low in $\mathrm{P}$, low in vitamin $\mathrm{D}$, and also low in sugar, does not coincide with many present-day theories regarding dietary control of dental disease. Furthermore, the production of caries by addition of a considerable quantity of candy to the diet of individuals known to be receiving an adequate balanced ration is very suggestive. The manner or extent in which sugar enters the problem of dental caries is not as yet apparent. It was found that, in a majority, adequate well-balanced diet, low in sugar, was followed by arrest of active caries, but that no single dietary principle applies equally to all persons.

During the course of this study it became very evident that heredity or individual characteristics may definitely determine caries susceptibility, irrespective of diet. Some caries-free individuals could eat any food, and any quantity of sugar, without resultant increase in dental caries or in B. acidophilus in the mouth. In others who were caries-susceptibles, the disease could not be checked by adherence to any ordinary satisfactory diet. A diet low in both starch and sugar invariably produced marked and rapid decrease in $\mathbf{B}$. acidophilus in the mouth. The number of individuals showing this inherent tendency to caries immunity or high susceptibility was small. 
In a great majority, B. acidophilus and dental caries were definitely changed by regulation of diet. These inherent tendencies of individuals should be taken into account in evaluating effects of any dietary program.

Summary. The findings of the Michigan Group on dental caries may be summarized as follows:

1. No consistent correlation could be found between amounts of salivary total solids, ash, $\mathrm{Ca}, \mathrm{P}, \mathrm{Cl}$, diastatic activity, $\mathrm{pH}, \mathrm{CO}_{2}$-capacity, or total alkalinity, and activity of dental caries.

2. No relationship could be found between intake of $\mathrm{Ca}, \mathrm{P}$, and vitamin $\mathrm{D}$, or acid-base dietary values, and activity of dental caries.

3. Evidence is submitted indicating that sugar is a very important consideration in dental caries.

4. A remarkably low degree of dental caries was observed in children on a low-sugar diet deficient in $\mathrm{Ca}, \mathrm{P}$, and vitamin $\mathrm{D}$.

5. By increasing sugar intake, active dental caries was induced in children while they were receiving a diet that was nutritionally adequate.

6. Ingestion of low-sugar diets was conducive to freedom from dental caries in a majority of individuals.

7. The most constant differential between caries-free and cariessusceptible individuals is that of relative number of $\mathrm{B}$. acidophilus organisms in the mouth. This correlation was approximately 90 percent positive.

8. There is a possible immunologic factor antagonistic to B. acidophilus in the blood of caries-free individuals, in whose mouths, as a rule, B. acidophilus does not exist; and when planted therein, promptly disappears.

9. The possibility of increasing immunity against B. acidophilus in caries-susceptible individuals by vaccine therapy is suggested.

10. Inherited tendencies or inherent individual characteristics were, in a small percentage of cases, more important determinant factors in caries susceptibility than ordinary dietary conditions. A great majority of caries-susceptible individuals, however, can apparently be benefited by simple dietary measures. 


\section{REFERENCES TO LITERATURE}

(1) Philip Jay, Mary Crowley, Faith Hadley, and R. W. Bunting. Bacteriologic and immunologic studies on dental caries. Jour. Amer. Den. Assoc, 20: 2130 (1933).

(2) Philip Jay, Mary Crowtey, and R. W. Buntrig. Preliminary studies on immunology of dental caries. Jour. Amer. Den. Assoc., 19: 265 (1932).

(3) Faith Hadley, R. W. Bunting, and Edna Delves. Recognition of bacillus acidophilus associated with dental caries; a preliminary report. Jour. Amer. Den. Assoc., 17: 2041 (1930).

(4) Faith Hadley and R. W. Bunting. Further studies on the recognition of B. acidophilus. Jour. Amer. Den. Assoc., 19: 28 (1932).

(5) Farth Hadley. A quantitative method for estimating Bacillus acidophilus in saliva. J. Dent. Res., 15: 415 (1933).

(6) Rebecca Hubbell and R. W. Bunting. Calcium and phosphorus of saliva in relation to dental caries. J. Nutrition, 5: 599 (1932).

(7) Rebecca Hubbell. The chemical composition of saliva and blood serum of children in relation to dental caries. Am. J. Physiology, 105: 436 (1933).

(8) Rebecca Hubbell and Martha Koenne. The effect of varying levels of sugar intake on nitrogen, calcium, and phosphorus retention of growing children. Am. J. Diseases of Children (in press).

(9) J. D. Boyd, C. L. Drain, ANd G. Stearns. Metabolic studies in children with dental caries. J. Biol. Chem., 103: 327 (1933).

(10) Martha Koenne, R. W. Bunting, and Elise Morrell. Studies in the control of dental caries in children. I. Am. J. Diseases of Children (in press).

(11) Martha KokHne and ElISe Morrell. The food requirements of girls six to thirteen years old. Ibid. (in press).

(12) Martha Koemne And R. W. Bunting. Studies in the control of dental caries. II. $J$. Nutrition (in press).

(13) Martha Koemne, R. W. Bunting, and Faith P. Hadiey. A review of recent studies of the cause of dental caries. J. Am. Diet. Assn., 9: 445 (1934). 